Pacific Journal of Mathematic 


\title{
NONCOMPACT, MINIMAL REGULAR SPACES
}

\author{
Manuel P. Berriozabal, Hon-Fei LaI \\ AND Dix H. PetTey
}

In the first known example of a noncompact, minimal regular space, the standard Tychonoff plank is used in the construction. In the present paper, this construction is generalized by using, instead of the standard Tychonoff plank, a generalized Tychonoff plank which is defined in terms of an arbitrary pair of ordinals. Such a construction yields a noncompact, minimal regular space if and only if both ordinals are limit ordinals and at least one of them is uncountably cofinal.

1. Introduction. Throughout this paper, $\omega$ and $\Omega$ shall denote, respectively, the first infinite ordinal and the first uncountable ordinal. For an arbitrary ordinal $\alpha$, we shall let $\alpha^{\prime}$ denote the set of all ordinals less than or equal to $\alpha$, with the order topology.

In [2], Berri and Sorgenfrey used countably infinitely many copies of the deleted Tychonoff plank $\omega^{\prime} \times \Omega^{\prime}-\{(\omega, \Omega)\}$ to construct a noncompact, minimal regular space. A natural speculation might be that the same construction could be used with any ordinals in place of $\omega$ and $\Omega$, or possibly any limit ordinals or any two limit ordinals of different cardinality. However, it will be shown here that such a construction yields a noncompact, minimal regular space if and only if both ordinals are limit ordinals and at least one of them is uncountably cofinal.

It is noted here that other techniques of construction-for example, extension spaces-can be used to obtain noncompact, minimal regular spaces. For a discussion of such techniques, the reader is referred to [1], [3], [5], [7] and [9]. For further discussion of results concerning limit ordinal numbers, the reader is referred to [4], [6] and [8].

Finally, it is noted that the principal result of this paper was obtained simultaneously by the first two authors, working together, and the third author, working independently.

2. The generalized Tychonoff plank. The Tychonoff plank $\omega^{\prime} \times \Omega^{\prime}$ has been the source of many counter-examples in general topology. For example, the Tychonoff plank with the point $(\omega, \Omega)$ removed is completely regular but not normal. In this section, we shall define generalized Tychonoff planks and prove a result, concerning these spaces, which will be used in $\S 3$. 
Let $\alpha$ and $\beta$ be ordinals greater than or equal to 2. The product $\alpha^{\prime} \times \beta^{\prime}$ is called a generalized Tychonoff plank. For the remainder of this paper, we fix $\alpha$ and $\beta \geqq 2$ and let $P$ denote $\alpha^{\prime} \times \beta^{\prime}-\{(\alpha, \beta)\}$. Obviously, $P$ is compact if and only if neither $\alpha$ nor $\beta$ is a limit ordinal. It is also obvious that $P$ is Hausdorff and regular.

Definition 2.1. A limit ordinal $\alpha$ is said to be countably cofinal if there exists an increasing sequence $\left\{\xi_{i}\right\}_{i=1}^{\infty}$ of ordinals $<\alpha$ converging to $\alpha$. Otherwise $\alpha$ is uncountably cofinal.

Definition 2.2. A subset $U$ of $P$ is said to get into the corner if, whenever $\xi_{0}<\alpha$ and $\eta_{0}<\beta$, there is a point $(\xi, \eta) \in U$ with $\xi_{0} \leqq$ $\xi<\alpha$ and $\eta_{0} \leqq \eta<\beta$. If both $\alpha, \beta$ are limit ordinals, this means that the corner $(\alpha, \beta)$ is a limit point of $U$ in $\alpha^{\prime} \times \beta^{\prime}$.

Proposition 2.1. Assume that both $\alpha, \beta$ are limit ordinals and at least one of them is uncountably cofinal. Let $U$ be an open set in $P$ which gets into the corner, and let $V$ be an open set containing the closure $\bar{U}$ of $U$. Then there exist $\xi_{0}<\alpha$ and $\eta_{0}<\beta$ such that $\bar{V}$ contains both the intervals $A=\left\{(\xi, \beta): \xi_{0} \leqq \xi<\alpha\right\}$ and $\beta=\{(\alpha, \eta)$ : $\left.\eta_{0} \leqq \eta<\beta\right\}$.

Proof. Without loss of generality, we can assume that $\beta$ is uncountably cofinal. Assume that either $\xi_{0}$ or $\eta_{0}$ does not exist. Then either the set $C=\{\xi: \xi<\alpha$ and $(\xi, \beta) \notin \bar{V}\}$ has $\beta$ as a limit point, or the set $D=\{\eta: \eta<\beta$ and $(\alpha, \eta) \notin \bar{V}\}$ has $\alpha$ as a limit point. In the first case, for each $\xi \in C$ there is $\lambda(\xi)<\beta$ such that, whenever $\lambda(\xi) \leqq \eta<\beta$ then $(\xi, \eta) \notin V$. Similarly in the second case. In each case, we conclude that $P-V$ gets into the corner. Since $U$ gets into the corner, we can then find two increasing sequences $\left\{\xi_{i}\right\}_{i=1}^{\infty}$ and $\left\{\eta_{i}\right\}_{i=1}^{\infty}$, with $\xi_{i}<\alpha$ and $\eta_{i}<\beta$, such that (a) for $i$ odd, $\left(\xi_{i}, \eta_{i}\right) \in U$, (b) for $i$ even, $\left(\xi_{i}, \eta_{i}\right) \notin V$. The sequences $\left\{\xi_{i}\right\},\left\{\eta_{i}\right\}$ have limits $\bar{\xi} \leqq \alpha$ and $\bar{\eta} \leqq \beta$ respectively. Since $\beta$ is uncountably cofinal, we must have $\bar{\eta}<\beta$. Then $(\bar{\xi}, \bar{\eta})$ is a point in $P$ lying in $\bar{U}$ but not in $V$, a contradiction.

REMARK. The full force of this proposition is not used in the following, but it gives a good insight into the structure of the generalized Tychonoff plank, and may be useful in the construction of other counter-examples.

3. The generalized extended Tychonoff construction. Let $J$ be the set of all integers, and consider the space $J \times \alpha^{\prime} \times \beta^{\prime}-$ $\{(n, \alpha, \beta): \eta \in J\}$ with the topology induced from $J \times \alpha^{\prime} \times \beta^{\prime}$. From 
this space, the quotient space $Y$ is defined in the following way: for even $n$, identify $(n, \alpha, \eta)$ with $(n+1, \alpha, \eta)$; for odd $n$, identify $(n, \xi, \beta)$ with $(n+1, \xi, \beta)$. We will continue to use the symbols $(n, \xi, \eta)$ for the points of $Y$; thus $(n, \alpha, \eta)=(n+1, \alpha, \eta)$ when $n$ is even. For $n \in J$, let $Q_{n}=\{(n, \xi, \eta): \xi<\alpha, \eta<\beta\}$ and $Z_{n}=\{(n, \xi, \eta)$ : $(\xi, \eta) \neq(\alpha, \beta)\}$. Let $p$ and $q$ be points not in $Y$ and topologize $Z=$ $\{p\} \cup\{q\} \cup Y$ by letting an open base at $p$ be all sets of the form

$$
V_{n}(p)=\cup\left\{Z_{i}: i>n\right\} \cup Q_{n} \cup\{p\}, \quad n=1,2, \cdots
$$

and an open base at $q$, all sets of the form

$$
V_{n}(q)=\cup\left\{Z_{-i}: i>n\right\} \cup Q_{-n} \cup\{q\}, \quad n=1,2, \cdots
$$

while all other points of $Y$ have as open bases those they had in the original quotient topology. Let $(Z, \mathscr{T})$ denote the resulting topological space, which will be called a generalized extended Tychonoff space. It is easily seen that $(Z, \mathscr{T})$ is always Hausdorff and regular. If $\alpha$ is the first infinite ordinal number and $\beta$ is the first uncountable ordinal number, then $(Z, \mathscr{T})$ is the noncompact, minimal regular space constructed in [2].

We say that a subset $U$ of $Z$ gets into the $n$-corner if $U \cap Z_{n}$ gets into the corner of $Z_{n}$. The main theorem of this paper is the following result.

THEOREM. $(Z, \mathscr{T})$ is noncompact and minimal regular if and only if both $\alpha$ and $\beta$ are limit ordinals and at least one of them is uncountably cofinal.

The proof makes use of the characterization (see [2]) of a minimal regular space as a regular space in which every regular filterbase with a unique adherent point is convergent. The proof also makes use of the property that, in a minimal regular space, every regular filter-base eas an adherent point.

Lemma 3.1. Assume that both $\alpha$ and $\beta$ are limit ordinals and that at least one of them is uncountably cofinal. Let $U, V, W$ be open sets of $Z$ such that $U \subset \bar{U} \subset V \subset \bar{V} \subset W$. If $U$ gets into the $n$-corner, then $W$ gets into the $(n-1)$-corner and $(n+1)$-corner.

Proof. We will prove for $n$ even; the proof for $n$ odd is similar. By Proposition 2.1, $\bar{V}$ contains an interval $\left\{(\xi, \beta): \xi_{0} \leqq \xi<\alpha\right\}$ for some $\xi_{0}$. Then, whenever $\xi_{0} \leqq \xi<\alpha,(n-1, \xi, \beta)=(n, \xi, \beta) \in \bar{V} \subset W$. $W$ being open, this implies that $W$ gets into the $(n-1)$-corner. Similarly $W$ gets into the $(n+1)$-corner. 


\section{Proof of Theorem.}

Sufficiency. If we make use of Lemma 3.1, the proof that $(Z, \mathscr{T})$ is minimal regular is identical to the corresponding part of the proof in [2]. Furthermore, $(Z, \mathscr{T})$ is not compact since the closed subspace $\{(0, \alpha, \eta): \eta<\beta\}$ is not compact.

Necessity. Assume that one of $\alpha$ and $\beta$ is not a limit ordinal or that both $\alpha$ and $\beta$ are countably cofinal. We will show that $(Z, \mathscr{T})$ is either compact or not minimal regular. We consider the following cases.

(i) Neither $\alpha$ nor $\beta$ is a limit ordinal. Then for each $n \in J$, $Z_{n}$ is a compact subspace of $Z$, thus $Y$ is locally compact and $(Z, \mathscr{T})$ is compact.

(ii) Exactly one of $\alpha, \beta$ is not a limit ordinal, say $\alpha$. For each $\eta<\beta$, let $F_{\eta}=\{(0, \alpha, \zeta): \eta<\zeta<\beta\}$. Then each $F_{\eta}$ is open and closed in $Z$, and the filter-base $\left\{F_{\eta}: \eta<\beta\right\}$ is a regular filter-base with no adherent point. Hence $(Z, \mathscr{T})$ is not minimal regular.

(iii) Both $\alpha, \beta$ are limit ordinals and are countably cofinal. Then there are increasing sequences $\left\{\xi_{k}\right\}_{k=1}^{\infty}$ and $\left\{\eta_{k}\right\}_{k-1}^{\infty}$ converging to $\alpha, \beta$ respectively. For each $m$, let $\left.F_{m}=\left(0, \xi_{k}+1, \eta_{k}+1\right): k \geqq m\right\}$. Each $F_{m}$ is open and closed, and the filter-base $\left\{F_{m}: m=1,2, \cdots\right\}$ is a regular filter-base with no adherent point. Thus $(Z, \mathscr{T})$ is not minimal regular.

We conclude the paper with the following remarks.

1. The example of a noncompact, minimal regular space in [2] satisfies the condition of the main theorem of this paper since $\omega$ is countably cofinal and $\beta$ is uncountably cofinal.

2. From the main theorem, $(Z, \mathscr{T})$ is minimal regular only if at least one of $\alpha$ or $\beta$ is uncountably cofinal and therefore uncountable. But mere uncountability is not sufficient. For example, if $\alpha=\Omega \omega \beta=\omega^{\Omega \omega}$, then both $\alpha$ and $\beta$ are uncountable ordinals and both are countably cofinal. Hence $(Z, \mathscr{T})$ is not minimal regular.

\section{REFERENCES}

1. M. P. Berri, J. R. Porter and R. M. Stephenson, J., A Survey of Minimal Topological Spaces, Proceedings of the Kanpur Topological Conference, (1968), 93-114.

2. M. P. Berri and R. H. Sorgenfrey, Minimal regular spaces, Proc. Amer. Math. Soc., 14 (1963), 454-458.

3. E. Čech and J. Novák, On regular and combinatorial imbedding, Časopes Pěst. Mat., 72 (1947), 7-16.

4. F. Hausdorff, Set Theory, 3rd Edition (translated), Chelsea, New York, (1957).

5. H. Herrlich, Regular-closed, Urysohn-closed and completely Hausdorff-closed spaces, Proc. Amer. Math. Soc., 26 (1970), 695-698.

6. I. Juhász, Cardinal Functions in Topology, Math. Center Tracts, North-Holland, 
Amsterdam, 1971.

7. J. Porter and C. Votaw, $S(\alpha)$ spaces and regular Hausdorff extensions, Pacific J. Math., 45 (1973), 327-345.

8. W. Sierpinski, Cardinal and Ordinal Numbers, Polska Biblio Analit Mech., Monografie Matematyozne, Warszawa, 1958. (English translation.)

9. R. M. Stephenson, Products of minimal Urysohn spaces, Duke Math. J., 38 (1971), 703-708.

Received April 30, 1976 and in revised form October 27, 1976.

UNIVERSITY OF NEW ORLEANS

The University of Texas at San Antonio

FORDHAM UNIVERSITY

AND

UNIVERSITY OF MisSOURI AT COLUMBIA 



\section{PACIFIC JOURNAL OF MATHEMATICS}

EDITORS

RICHARD ARENS (Managing Editor)

University of California

Los Angeles, California 90024

R. A. Beaumont

University of Washington

Seattle, Washington 98105
J. DUGUNDJI

Department of Mathematics

University of Southern Californı

Los Angeles, California 90007

D. Gilbarg and J. Milgram

Stanford University

Stanford, California 94305

\section{ASSOCIATE EDITORS}

E. F. BECKENBACH

B. H. NeumanN

F. WOLF

K. YoSHIDA

\section{SUPPORTING INSTITUTIONS}

UNIVERSITY OF BRITISH COLUMBIA

CALIFORNIA INSTITUTE OF TECHNOLOGY

UNIVERSITY OF CALIFORNIA

MONTANA STATE UNIVERSITY

UNIVERSITY OF NEVADA

NEW MEXICO STATE UNIVERSITY

OREGON STATE UNIVERSITY

UNIVERSITY OF OREGON

OSAKA UNIVERSITY
UNIVERSITY OF SOUTHERN CALIFORNIA

STANFORD UNIVERSITY

UNIVERSITY OF TOKYO

UNIVERSITY OF UTAH

WASHINGTON STATE UNIVERSITY

UNIVERSITY OF WASHINGTON

AMERICAN MATHEMATICAL SOCIETY

NAVAL WEAPONS CENTER 


\section{Pacific Journal of Mathematics}

Vol. 69 , No. 1

May, 1977

V. V. Anh and P. D. Tuan, On starlikeness and convexity of certain analytic

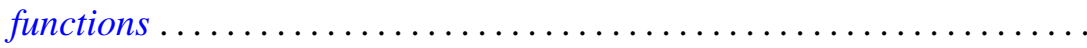

Willard Ellis Baxter and L. A. Casciotti, Rings with involution and the prime

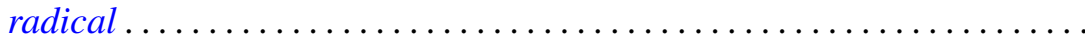

Manuel Phillip Berriozabal, Hon-Fei Lai and Dix Hayes Pettey,

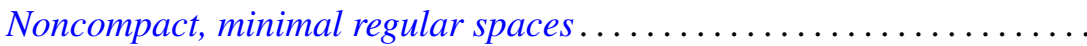

Sun Man Chang, Measures with continuous image law ................

John Benjamin Friedlander, Certain hypotheses concerning

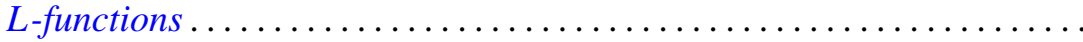

Moshe Goldberg and Ernst Gabor Straus, On characterizations and

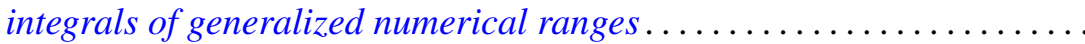

Pierre A. Grillet, On subdirectly irreducible commutative semigroups ...... 55

Robert E. Hartwig and Jiang Luh, On finite regular rings ..............

Roger Hugh Hunter, Fred Richman and Elbert A. Walker, Finite direct sums of cyclic valuated p-groups ........................... 97

Atsushi Inoue, On a class of unbounded operator algebras. III ......... 105

Wells Johnson and Kevin J. Mitchell, Symmetries for sums of the Legendre

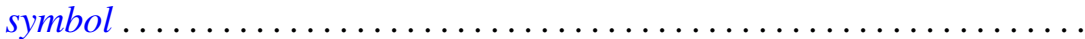

Jimmie Don Lawson, John Robie Liukkonen and Michael William Mislove,

Measure algebras of semilattices with finite breadth

Glenn Richard Luecke, A note on spectral continuity and on spectral properties of essentially $G_{1}$ operators ...............

Takahiko Nakazi, Invariant subspaces of weak-* Dirichlet algebras . .

James William Pendergrass, Calculations of the Schur group ...

Carl Pomerance, On composite $n$ for which $\varphi(n) \mid n-1$. II. . .

Marc Aristide Rieffel and Alfons Van Daele, A bounded operator approach to Tomita-Takesaki theory........................

Daniel Byron Shapiro, Spaces of similarities. IV. $(s, t)$-families ...

Leon M. Simon, Equations of mean curvature type in 2 independent variables.

Joseph Nicholas Simone, Metric components of continuous images of ordered compacta ............................

William Charles Waterhouse, Pairs of symmetric bilinear forms in

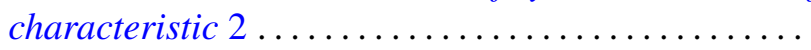

